\title{
Generalization of Statistical Korovkin Theorems
}

\author{
Alperen Ali Ergur and Oktay Duman \\ Department of Mathematics, TOBB Economics and Technology University, Söğütözü, 06530 Ankara, Turkey
}

Correspondence should be addressed to Oktay Duman; oduman@etu.edu.tr

Received 18 March 2013; Accepted 21 May 2013

Academic Editor: Juan Manuel Peña

Copyright (C) 2013 A. A. Ergur and O. Duman. This is an open access article distributed under the Creative Commons Attribution License, which permits unrestricted use, distribution, and reproduction in any medium, provided the original work is properly cited.

We generalize and develop the Korovkin-type approximation theory by using an appropriate abstract space. We show that our approximation is more applicable than the classical one. At the end, we display some applications.

\section{Introduction}

The classical Korovkin theory enables us to approximate a function by means of positive linear operators (see, e.g., [13]). In recent years, this theory has been quite improved by some efficient tools in mathematics such as the concept of statistical convergence from summability theory, the fuzzy logic theory, the complex functions theory, the theory of $q$-calculus, and the theory of fractional analysis. The main purpose of this paper is to generalize and develop this Korovkin theory by using an appropriate abstract space. Actually, the most important motivation of this study has its roots from the paper by Yoshinaga and Tamura [4]. In the present paper, we show that our new approximation is more general and also more applicable than that of [4].

Throughout the paper the following assumptions are imposed:

(i) $(X, \mathcal{U})$ is a Hausdorff uniform space provided with the uniform structure $(X, \mathcal{U})$;

(ii) $\mathcal{U}$ is the filter of the surroundings containing the diagonal $\Delta=\{(x, x): x \in X\}$ in $X \times X$;

(iii) $\mathscr{F}$ is a vector space of real-valued functions defined on $X$ including the constant-valued function $e_{0}(x)=$ 1 ;

(iv) $Y$ is a compact subspace of $X$;

(v) $L_{n}$ is a positive linear operator of $\mathscr{F}$ into $\mathbb{R}^{Y}$ for each $n \in \mathbb{N}$;

(vi) $A:=\left[a_{j n}\right]$ is a nonnegative regular summable matrix.
Assume further that there exists a certain real-valued function $F(x, y)$ satisfying the following conditions:

(i) $F(x, y) \geq 0$ on $X \times Y$ and $F(y, y)=0$ for each $y \in Y$;

(ii) $F_{y} \in \mathscr{F}$ for each $y \in Y$, where $F_{y}$ is the function on $X$ defined by $F_{y}(x):=F(x, y)$;

(iii) for each $y \in Y, F_{y}(x)$ is continuous with respect to $x$ at each point in $Y$;

(iv) $\rho(U)=\inf F(x, y)>0$ for each $U \in \mathcal{U}$, where the infimum is taken over $(X \times Y)-U$;

(v) there exist $y_{1}, y_{2} \in Y, y_{1} \neq y_{2}$, such that $F_{y_{1}}(x)$ and $F_{y_{2}}(x)$ are bounded functions of $x$, and it holds that

$$
\begin{aligned}
& \mathrm{st}_{A}-\lim _{n}\left\|L_{n}\left(F_{y_{1}}\right)-F_{y_{1}}\right\|=0, \\
& \mathrm{st}_{A}-\lim _{n}\left\|L_{n}\left(F_{y_{2}}\right)-F_{y_{2}}\right\|=0, \\
& \mathrm{st}_{A}-\lim _{n}\left(\sup _{y \in Y} L_{n}\left(F_{y} ; y\right)\right)=0,
\end{aligned}
$$

where the symbol $\|\cdot\|$ denotes the classical sup-norm on the compact set $Y$. Here, we use the concept of $A$-statistical convergence, where $A$ is a nonnegative regular summable matrix. Recall that, for a given subset $K$ of $\mathbb{N}$, the $A$-density of $K$, denoted by $\delta_{A}(K)$, is defined to be $\delta_{A}(K)=\lim _{j \rightarrow \infty} \sum_{n \in K} a_{j n}$ provided that the limit exists. Using this $A$-density, we say that a sequence $x=\left(x_{n}\right)$ is $A$-statistically convergent to $L$ if and only if $\delta_{A}(K(\varepsilon))=0$ for every $\varepsilon>0$, where $K(\varepsilon):=\{n \in \mathbb{N}$ : $\left.\left|x_{n}-L\right| \geq \varepsilon\right\}$ (see [5]). In the case of $A=C_{1}$, the Cesàro matrix, 
it reduces to the concept of statistical convergence introduced by Fast [6]. Of course, if we take $A=I$, the identity matrix, then we get the ordinary convergence.

We should note that if, for each $y \in Y, F_{y}(x)$ is a bounded function of $x$ for which

$$
\mathrm{st}_{A}-\lim _{n}\left(\sup _{x, y \in Y}\left|L_{n}\left(F_{y} ; x\right)-F_{y}(x)\right|\right)=0
$$

holds, then we get the conditions in $(v)$.

Then, with the above terminology, Yoshinaga and Tamura [4] proved the following approximation result (in the case of $A=I)$.

Theorem A (see [4]). Let $f$ be a bounded real-valued function on $X$ and continuous at each point in $Y$. Then, if $f \in \mathscr{F}$, the sequence $\left\{L_{n}(f)\right\}$ is uniformly convergent to $f$ on $Y$.

\section{Statistical Approximation Theorem}

In this section, we obtain the statistical analog of Theorem A in order to get a more applicable approximation theorem.

We first need the following three lemmas.

Lemma 1 (see [4]). Let $V$ be an open subset of $X \times Y$ containing $\Delta_{Y}:=\Delta \cap(X \times Y)$. Then, it is possible to see that $U \cap(X \times Y) \subset V$ for some $U \in \mathcal{U}$.

Lemma 2. The sequence $\left\{\left\|L_{n}\left(e_{0}\right)\right\|\right\}$ is A-statistically bounded; that is; there exist a positive real number $M$ and a subset $K \subset \mathbb{N}$ having A-density 1 such that

$$
\left\|L_{n}\left(e_{0}\right)\right\| \leq M \quad \text { for every } n \in K .
$$

Proof. For the points $y_{1}, y_{2}$ given in $(v)$, we can take $U_{0} \in \mathcal{U}$ so that $\left(y_{1}, y_{2}\right) \notin U_{0}$. Now, choose $U \in \mathcal{U}$ such that $U=U^{-1}$ and $U \circ U \subset U_{0}$. Then, we observe, for every $x \in X$, that

$$
F_{y_{1}}(x)+F_{y_{2}}(x) \geq \rho(U) .
$$

Hence, we get, for each $y \in Y$ and for every $n \in \mathbb{N}$, that

$$
L_{n}\left(F_{y_{1}} ; y\right)+L_{n}\left(F_{y_{2}} ; y\right) \geq \rho(U) L_{n}\left(e_{0} ; y\right) \geq 0,
$$

which implies that

$$
\begin{aligned}
\rho(U) L_{n}\left(e_{0} ; y\right) \leq & \left|L_{n}\left(F_{y_{1}} ; y\right)-F_{y_{1}}(y)\right| \\
& +\left|L_{n}\left(F_{y_{2}} ; y\right)-F_{y_{2}}(y)\right| \\
& +\left\{F_{y_{1}}(y)+F_{y_{2}}(y)\right\} .
\end{aligned}
$$

Taking supremum over $y \in Y$ and also letting

$$
M_{1}:=\frac{1}{\rho(U)}\left\|F_{y_{1}}+F_{y_{2}}\right\|,
$$

we obtain, for every $n \in \mathbb{N}$, that

$$
\begin{aligned}
\left\|L_{n}\left(e_{0}\right)\right\| \leq & \frac{1}{\rho(U)}\left\|L_{n}\left(F_{y_{1}}\right)-F_{y_{1}}\right\| \\
& +\frac{1}{\rho(U)}\left\|L_{n}\left(F_{y_{2}}\right)-F_{y_{2}}\right\|+M_{1} .
\end{aligned}
$$

Now, for a given $\varepsilon>0$, define the following sets:

$$
\begin{aligned}
& K_{1}:=\left\{n \in \mathbb{N}:\left\|L_{n}\left(F_{y_{1}}\right)-F_{y_{1}}\right\| \geq \frac{\varepsilon \rho(U)}{2}\right\}, \\
& K_{2}:=\left\{n \in \mathbb{N}:\left\|L_{n}\left(F_{y_{2}}\right)-F_{y_{2}}\right\| \geq \frac{\varepsilon \rho(U)}{2}\right\} .
\end{aligned}
$$

Then, from the conditions in $(v)$, we may write that

$$
\delta_{A}\left(K_{1}\right)=\delta_{A}\left(K_{2}\right)=0 .
$$

Now setting

$$
K:=\mathbb{N}-\left(K_{1} \cup K_{2}\right),
$$

we immediately get that

$$
\delta_{A}(K)=1 .
$$

Furthermore, it follows from (8) that, for every $n \in K$, that is, $n \notin K_{1}$ and $n \notin K_{2}$,

$$
\left\|L_{n}\left(e_{0}\right)\right\| \leq \varepsilon+M_{1}=: M,
$$

which completes the proof.

Lemma 3. Let $g(x, y)$ be a real-valued and bounded function on $X \times Y$, and let $g$ be continuous at each diagonal point $(y, y) \in \Delta_{Y}$. For each $y \in Y$, define the function $g_{y}$ on $X$ by $g_{y}(x):=g(x, y)$. Assume further that $g_{y} \in \mathscr{F}$ and $g_{y}(y)=0$ for each $y \in Y$. Then, one has

$$
s t_{A}-\lim _{n}\left(\sup _{y \in Y}\left|L_{n}\left(g_{y} ; y\right)\right|\right)=0 .
$$

Proof. Since $g$ is continuous at any diagonal point $(y, y) \epsilon$ $\Delta_{Y}$, for every $\varepsilon>0$, there exists an open neighborhood $V(y)$ of $y$ in $X$ such that

$$
|g(x, y)|=|g(x, y)-g(y, y)|<\varepsilon
$$

for every $(x, y) \in V(y) \times(V(y) \cap Y)$. Now, if we define the set $V$ by

$$
V:=\bigcup_{y \in Y}\{V(y) \times(V(y) \cap Y)\}
$$

then we easily see that $V$ is an open subset of $X \times Y$ containing the diagonal $\Delta_{Y}$. Also, it follows from Lemma 1 that $U \cap(X \times$ $Y) \subset V$ for some $U \in \mathcal{U}$. Now, setting

$$
C:=\sup _{(x, y) \in X \times Y}|g(x, y)|,
$$

we get, for every $(x, y) \in X \times Y$, that

$$
|g(x, y)| \leq \varepsilon+\frac{C}{\rho(U)} F_{y}(x),
$$

which in turn implies that

$$
\begin{aligned}
\left|L_{n}\left(g_{y} ; y\right)\right| & \leq L_{n}\left(\left|g_{y}\right| ; y\right) \\
& \leq \varepsilon L_{n}\left(e_{0} ; y\right)+\frac{C}{\rho(U)} L_{n}\left(F_{y} ; y\right) .
\end{aligned}
$$


Thus we conclude that, for every $n \in \mathbb{N}$, the inequality

$$
\sup _{y \in Y}\left|L_{n}\left(g_{y} ; y\right)\right| \leq \varepsilon\left\|L_{n}\left(e_{0}\right)\right\|+\frac{C}{\rho(U)} \sup _{y \in Y} L_{n}\left(F_{y} ; y\right)
$$

holds. By Lemma 2, there exists a positive real number $M$ and a subset $K$ of $\mathbb{N}$ having $A$-density 1 such that

$$
\sup _{y \in Y}\left|L_{n}\left(g_{y} ; y\right)\right| \leq M \varepsilon+\frac{C}{\rho(U)} \sup _{y \in Y} L_{n}\left(F_{y} ; y\right)
$$

holds for every $n \in K$. Now, for a given $r>0$, choose an $\varepsilon>0$ such that $r>M \varepsilon$. Then, considering the following subsets of $\mathbb{N}$ :

$$
\begin{aligned}
& D:=\left\{n \in \mathbb{N}: \sup _{y \in Y}\left|L_{n}\left(g_{y} ; y\right)\right| \geq r\right\} \\
& D^{\prime}:=\left\{n \in \mathbb{N}: \sup _{y \in Y} L_{n}\left(F_{y} ; y\right) \geq \frac{(r-\varepsilon M) \rho(U)}{C}\right\}
\end{aligned}
$$

and also using (21), we have $D \cap K \subset D^{\prime} \cap K \subset D^{\prime}$, which gives, for every $j \in \mathbb{N}$, that

$$
0 \leq \sum_{n \in D \cap K} a_{j n} \leq \sum_{n \in D^{\prime} \cap K} a_{j n} \leq \sum_{n \in D^{\prime}} a_{j n} .
$$

Taking limit as $j \rightarrow \infty$ in both sides of the last inequality and also using $(v)$, we obtain that

$$
\lim _{j} \sum_{n \in D \cap K} a_{j n}=0 .
$$

Furthermore, we may write that

$$
\begin{aligned}
\sum_{n \in D} a_{j n} & =\sum_{n \in D \cap K} a_{j n}+\sum_{n \in D \cap(\mathbb{N}-K)} a_{j n} \\
& \leq \sum_{n \in D \cap K} a_{j n}+\sum_{n \in(\mathbb{N}-K)} a_{j n} .
\end{aligned}
$$

Since $\delta_{A}(K)=1$, we get $\delta_{A}(\mathbb{N}-K)=0$. Thus, by (24) and (25), we obtain that

$$
\lim _{j} \sum_{n \in D} a_{j n}=0
$$

which means

$$
\mathrm{st}_{A}-\lim _{n}\left(\sup _{y \in Y}\left|L_{n}\left(g_{y} ; y\right)\right|\right)=0 .
$$

Therefore, the proof is completed.

Now we are ready to give our main approximation result in statistical sense.

Theorem 4. Let $f$ be a bounded real-valued function on $X$ and continuous at each point in $Y$. Then, if $f \in \mathscr{F}$, one has

$$
s t_{A}-\lim _{n}\left\|L_{n}(f)-f\right\|=0 .
$$

Proof. As in the proof of Lemma 2, we take $U_{0}, U \in \mathscr{U}$ such that $U=U^{-1},\left(y_{1}, y_{2}\right) \notin U_{0}$, and $U \circ U \subset U_{0}$, where $y_{1}, y_{2}$ are given in $(v)$. Let

$$
\begin{aligned}
& G(x):=F\left(x, y_{1}\right)+F\left(x, y_{2}\right), \\
& g(x, y):=f(x)-\frac{f(y)}{G(y)} G(x)
\end{aligned}
$$

for $(x, y) \in X \times Y$. Then, we see that $G \in \mathscr{F}$, and so $0<$ $\rho(U) \leq G(x) \leq C$ for some $C>0$ due to the boundedness of the functions $F_{y_{1}}$ and $F_{y_{2}}$ on $X$. Also, observe that $g_{y} \in \mathscr{F}$ and $g_{y}(y)=0$ for each $y \in Y$. Since $f$ and $G$ are continuous at any point $y \in Y$ and also $G(y) \geq \rho(U)>0$, we easily check that the function $g(x, y)$ is continuous at each point $(y, y) \in \Delta_{y}$. Since $f$ is bounded on $X$, we may write that $M:=\sup _{x \in X}|f(x)|<\infty$. Then, it is not hard to see that

$$
|g(x, y)| \leq M+\frac{M}{\rho(U)} C
$$

for every $(x, y) \in X \times Y$. From Lemma 3 , one can get that

$$
\operatorname{st}_{A}-\lim _{n}\left(\sup _{y \in Y}\left|L_{n}\left(g_{y} ; y\right)\right|\right)=0 .
$$

On the other hand, by (29), we have

$$
g_{y}(x)=f(x)-\frac{f(y)}{F_{y_{1}}(y)+F_{y_{2}}(y)}\left(F_{y_{1}}(x)+F_{y_{2}}(x)\right),
$$

which yields that

$$
\begin{aligned}
L_{n}\left(g_{y} ; y\right)= & L_{n}(f ; y) \\
& -\frac{f(y)}{F_{y_{1}}(y)+F_{y_{2}}(y)}\left(L_{n}\left(F_{y_{1}} ; y\right)+L_{n}\left(F_{y_{2}} ; y\right)\right) .
\end{aligned}
$$

Hence we get

$$
\begin{aligned}
L_{n}(f ; y) & -f(y) \\
= & L_{n}\left(g_{y} ; y\right)+\frac{f(y)}{F_{y_{1}}(y)+F_{y_{2}}(y)} \\
\quad \times & \left\{\left(L_{n}\left(F_{y_{1}} ; y\right)-F_{y_{1}}(y)\right)\right. \\
& \left.+\left(L_{n}\left(F_{y_{2}} ; y\right)-F_{y_{2}}(y)\right)\right\} .
\end{aligned}
$$

Taking supremum over $y \in Y$, we immediately obtain that

$$
\begin{aligned}
\left\|L_{n}(f)-f\right\| \leq \sup _{y \in Y}\left|L_{n}\left(g_{y} ; y\right)\right| & \\
+\frac{M}{\rho(U)}\{ & \left\{\left\|L_{n}\left(F_{y_{1}}\right)-F_{y_{1}}\right\|\right. \\
& \left.+\left\|L_{n}\left(F_{y_{2}}\right)-F_{y_{2}}\right\|\right\} .
\end{aligned}
$$


Now, for a given $\varepsilon>0$, define the following sets:

$$
\begin{gathered}
E:=\left\{n \in \mathbb{N}:\left\|L_{n}(f)-f\right\| \geq \varepsilon\right\}, \\
E_{1}:=\left\{n \in \mathbb{N}: \sup _{y \in Y}\left|L_{n}\left(g_{y} ; y\right)\right| \geq \frac{\varepsilon}{3}\right\}, \\
E_{2}:=\left\{n \in \mathbb{N}:\left\|L_{n}\left(F_{y_{1}}\right)-F_{y_{1}}\right\| \geq \frac{\varepsilon \rho(U)}{3 M}\right\}, \\
E_{3}:=\left\{n \in \mathbb{N}:\left\|L_{n}\left(F_{y_{2}}\right)-F_{y_{2}}\right\| \geq \frac{\varepsilon \rho(U)}{3 M}\right\} .
\end{gathered}
$$

Then, it follows from (35) that

$$
E \subset E_{1} \cup E_{2} \cup E_{3},
$$

which guarantees that, for any $j \in \mathbb{N}$,

$$
\sum_{n \in E} a_{j n} \leq \sum_{n \in E_{1}} a_{j n}+\sum_{n \in E_{2}} a_{j n}+\sum_{n \in E_{3}} a_{j n} .
$$

Now letting $j \rightarrow \infty$ and also using $(v)$ and (31), we conclude that

$$
\lim _{j} \sum_{n \in E} a_{j n}=0
$$

which is the desired result.

\section{Concluding Remarks}

If we take $A=I$, the identity matrix, in Theorem 4 , then we easily get Theorem $A$. Hence, one can say that Theorem 4 covers Theorem A. However, if we take $A=C_{1}$, the Cesàro matrix, and also define the sequence $\left(u_{n}\right)$ by

$$
u_{n}:= \begin{cases}1, & n=m^{2}, \quad m \in \mathbb{N} \\ 0, & \text { otherwise }\end{cases}
$$

then we observe that

$$
\mathrm{st}_{C_{1}}-\lim _{n} u_{n}=\mathrm{st}-\lim _{n} u_{n}=0
$$

although it is nonconvergent in the usual sense. Now, assume that $\left\{L_{n}\right\}$ is a sequence of positive linear operators satisfying all conditions of Theorem A. Then, using $\left(u_{n}\right)$ and $\left(L_{n}\right)$, we construct new operators $T_{n}$ as follows:

$$
T_{n}(f):=\left(1+u_{n}\right) L_{n}(f) \text { for every } f \in \mathscr{F} .
$$

In this case, we verify that our operators $T_{n}$ satisfy all conditions of Theorem 4 due to property (41). Thus, we may write that, for every $f \in \mathscr{F}$,

$$
\text { st }-\lim _{n}\left\|T_{n}(f)-f\right\|=0 .
$$

However, since the sequence $\left(u_{n}\right)$ given by $(40)$ is nonconvergent, approximating a function $f \in \mathscr{F}$ by the operators $T_{n}$ is impossible. This example clearly shows that Theorem 4 is a nontrivial generalization of Theorem A.

Now we give some significant applications of Theorem 4 . As usual, by $C(X)$ we denote the space of all real-valued continuous functions on $X$.
Corollary 5 (see Theorem 3.5 of [7]). Let $X$ be a compact Hausdorff space, and let $f_{1}, f_{2}, \ldots, f_{m} \in C(X)$ satisfy the condition that there exist $g_{1}, g_{2}, \ldots, g_{m} \in C(X)$ such that defining $P(x, y):=\sum_{i=1}^{m} g_{i}(y) f_{i}(x)$ for every $x, y \in X$, it holds that $P(x, y) \geq 0$ and $P(x, y)=0$ if and only if $x=y$. Assume that $\left\{L_{n}\right\}$ is a sequence of positive linear operators from $C(X)$ into itself. Assume further that, for a given nonnegative regular summable matrix $A=\left[a_{j n}\right]$,

$$
s t_{A}-\lim _{n}\left\|L_{n}\left(f_{i}\right)-f_{i}\right\|=0 \quad \text { for each } i=1,2, \ldots, m .
$$

Then, for every $f \in C(X)$, one has $s t_{A}-\lim _{n}\left\|L_{n}(f)-f\right\|=0$.

Proof. Take $\mathscr{F}=C(X), X=Y$, and $F(x, y)=P(x, y)$. Then, since

$$
L_{n}\left(P_{y} ; x\right)-P_{y}(x)=\sum_{i=1}^{m} g_{i}(y)\left\{L_{n}\left(f_{i} ; x\right)-f_{i}(x)\right\},
$$

we observe that

$$
\sup _{x, y \in X}\left|L_{n}\left(P_{y} ; x\right)-P_{y}(x)\right| \leq C \sum_{i=1}^{m}\left\|L_{n}\left(f_{i}\right)-f_{i}\right\|,
$$

where $C:=\max _{1 \leq i \leq m}\left\|g_{i}\right\|$. Now, for a given $\varepsilon>0$, consider the following sets:

$$
\begin{aligned}
& B:=\left\{n \in \mathbb{N}: \sup _{x, y \in X}\left|L_{n}\left(P_{y} ; x\right)-P_{y}(x)\right| \geq \varepsilon\right\}, \\
& B_{i}:=\left\{n \in \mathbb{N}:\left\|L_{n}\left(f_{i}\right)-f_{i}\right\| \geq \frac{\varepsilon}{m C}\right\}, \quad i=1,2, \ldots, m .
\end{aligned}
$$

Hence, inequality (46) implies that

$$
B \subset \bigcup_{i=1}^{m} B_{i}
$$

which gives, for every $j \in \mathbb{N}$, that

$$
\sum_{n \in B} a_{j n} \leq \sum_{i=1}^{m} \sum_{n \in B_{i}} a_{j n}
$$

By (44), we obtain that

$$
\mathrm{st}_{A}-\lim _{n} \sum_{n \in B} a_{j n}=0,
$$

which gives

$$
\mathrm{st}_{A}-\lim _{n}\left(\sup _{x, y \in X}\left|L_{n}\left(P_{y} ; x\right)-P_{y}(x)\right|\right)=0 .
$$

Thus, the last equality means that condition (2) is valid for the function $P(x, y)$. As a result, all hypotheses of Theorem 4 are satisfied.

If we take $A=I$, the identity matrix, in Corollary 5 , then we immediately get the classical result (see, e.g., [3, page 22]).

In algebraic case, we consider the following test functions: $e_{i}(x)=x^{i}, i=0,1,2$. Then we get the next result. 
Corollary 6 (see Corollary 2 of [8]). Let $\left\{L_{n}\right\}$ be a sequence of positive linear operators from $C[a, b]$ into itself. If, for a given nonnegative regular summable matrix $A=\left[a_{j n}\right]$,

$$
s t_{A}-\lim _{n}\left\|L_{n}\left(e_{i}\right)-e_{i}\right\|=0 \quad \text { for each } i=0,1,2,
$$

then, for every $f \in C[a, b]$, one has st ${ }_{A}-\lim _{n}\left\|L_{n}(f)-f\right\|$.

Proof. If we take $X=\mathbb{R}, Y=[a, b], \mathscr{F}=C[a, b]$, and $F(x, y)=(y-x)^{2}$, then we observe that all conditions of Theorem 4 are satisfied.

Of course, if $A=C_{1}$, the Cesáro matrix, in Corollary 6, then one obtains Theorem 1 of [9]. Furthermore, taking $A=I$ we get the classical theorem (see [2]).

Finally, as in [4], Theorem 4 also contains the trigonometric version of Corollary 6 introduced in [10].

\section{Acknowledgments}

The authors would like to thank the referee for carefully reading the paper. The second author also thanks TUBA for their support.

\section{References}

[1] F. Altomare and M. Campiti, Korovkin-Type Approximation Theory and Its Applications, vol. 17 of de Gruyter Studies in Mathematics, Walter de Gruyter \& Co., Berlin, Germany, 1994.

[2] P. P. Korovkin, Linear Operators and Approximation Theory, Russian Monographs and Texts on Advanced Mathematics and Physics, Vol. III, Gordon and Breach, New York, NY, USA, 1960.

[3] H. N. Mhaskar and D. V. Pai, Fundamentals of Approximation Theory, CRC Press, Boca Raton, Fla, USA, 2000.

[4] K. Yoshinaga and S. Tamura, "On a Korovkin theorem of uniform convergence," Bulletin of the Kyushu Institute of Technology. Mathematics, Natural Science, no. 23, pp. 1-9, 1976.

[5] A. R. Freedman and J. J. Sember, "Densities and summability," Pacific Journal of Mathematics, vol. 95, no. 2, pp. 293-305, 1981.

[6] H. Fast, "Sur la convergence statistique," Colloquium Mathematicum, vol. 2, pp. 241-244, 1951.

[7] O. Duman and C. Orhan, "An abstract version of the Korovkin approximation theorem," Publicationes Mathematicae Debrecen, vol. 69 , no. 1-2, pp. 33-46, 2006.

[8] O. Duman, M. K. Khan, and C. Orhan, " $A$-statistical convergence of approximating operators," Mathematical Inequalities \& Applications, vol. 6, no. 4, pp. 689-699, 2003.

[9] A. D. Gadjiev and C. Orhan, "Some approximation theorems via statistical convergence," The Rocky Mountain Journal of Mathematics, vol. 32, no. 1, pp. 129-138, 2002.

[10] O. Duman, "Statistical approximation for periodic functions," Demonstratio Mathematica, vol. 36, no. 4, pp. 873-878, 2003. 


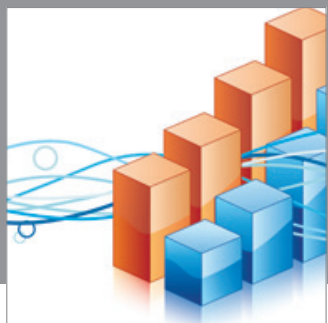

Advances in

Operations Research

mansans

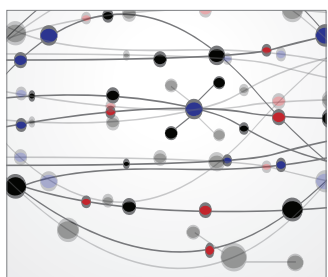

The Scientific World Journal
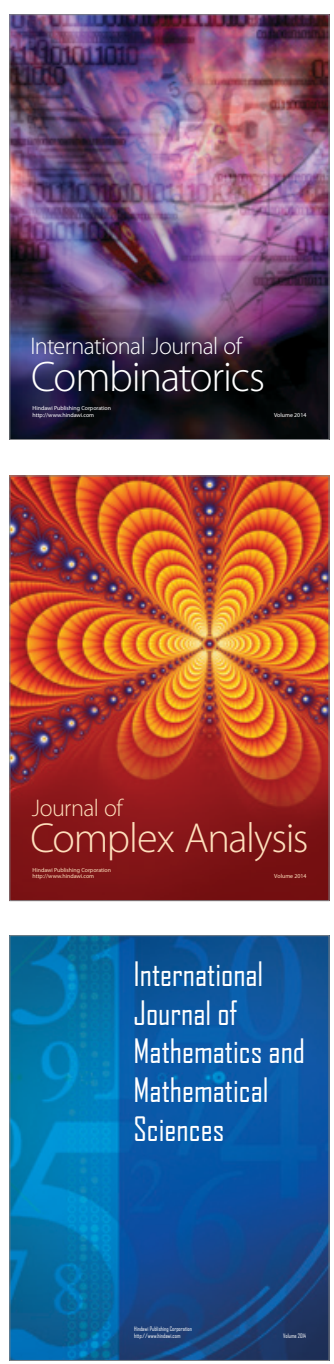
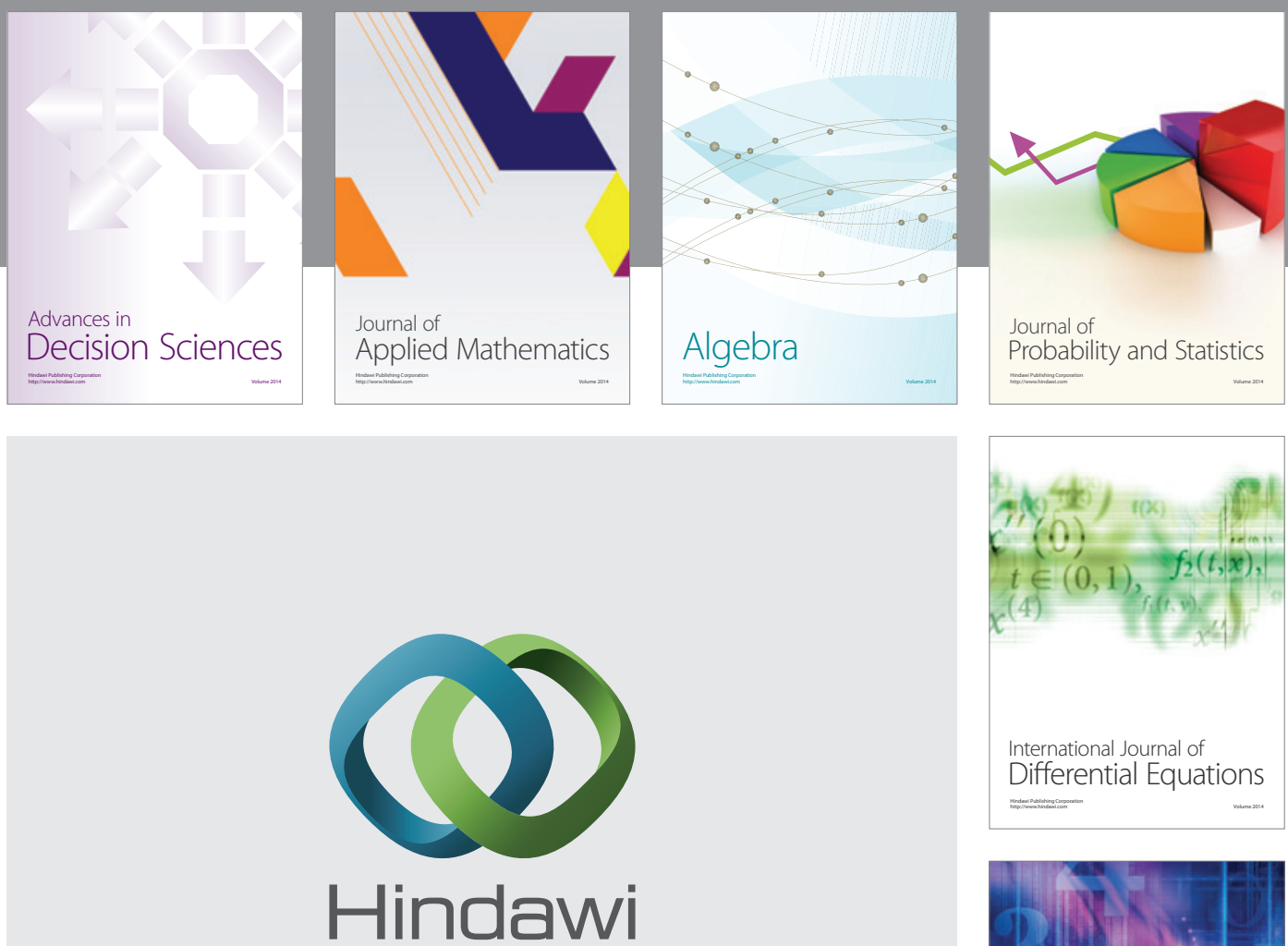

Submit your manuscripts at http://www.hindawi.com
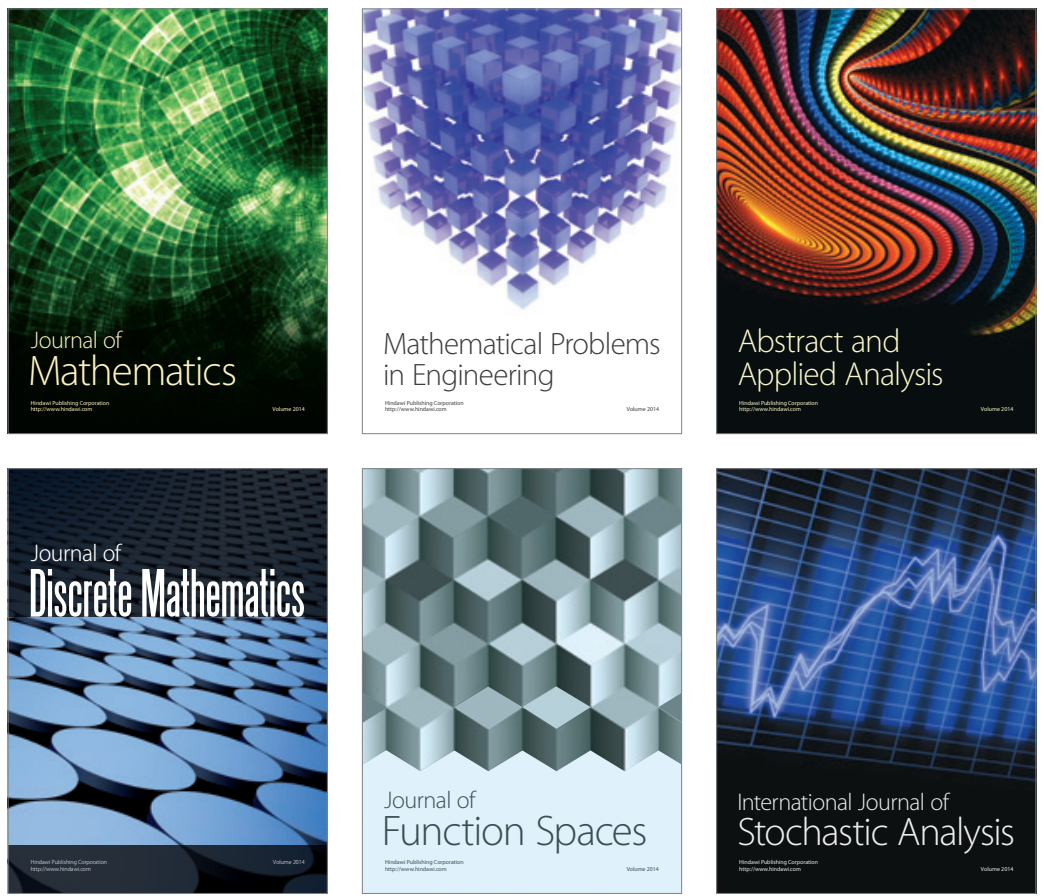

Journal of

Function Spaces

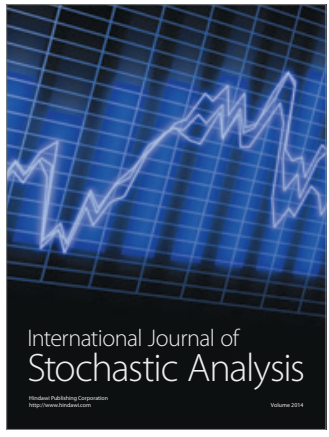

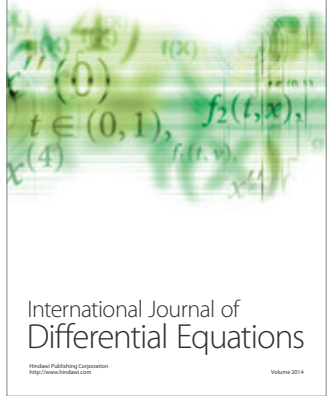
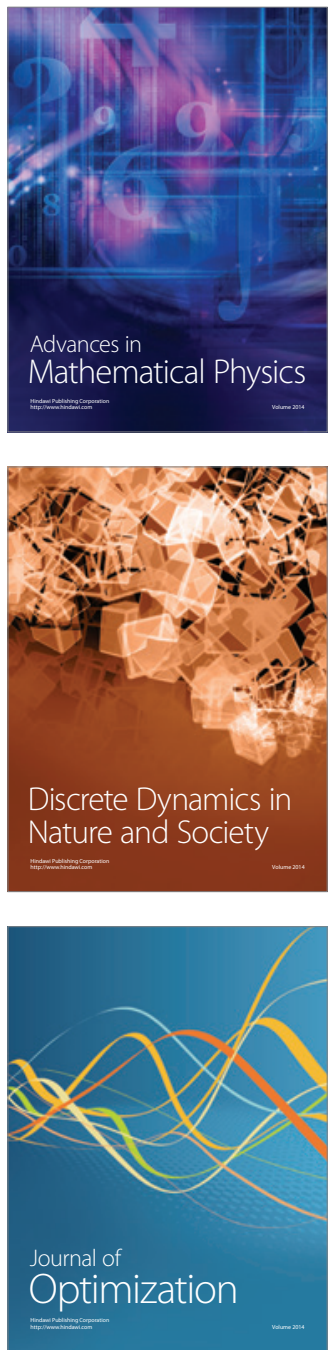